\title{
Observation of the effect of a 7-day gradual early functional exercise program in middle-aged and young patients with acute myocardial infarction after percutaneous coronary intervention
}

\author{
Yan Yang ${ }^{1}$, Lixing Sun ${ }^{1}$, Weijiao Feng ${ }^{2}$, Dan Sun ${ }^{3}$ \\ ${ }^{1}$ Department of Cardiology, the Affiliated Hospital of Jiangnan University, Wuxi, China; ${ }^{2}$ Department of Neurology, the Affiliated Hospital of \\ Jiangnan University, Wuxi, China; ${ }^{3}$ Department of Burns, the Affiliated Hospital of Jiangnan University, Wuxi, China \\ Contributions: (I) Conception and design: Y Yang, L Sun, W Feng; (II) Administrative support: D Sun; (III) Provision of study materials or patients: Y \\ Yang, L Sun; (IV) Collection and assembly of data: Y Yang, W Feng; (V) Data analysis and interpretation: Y Yang, L Sun, W Feng; (VI) Manuscript \\ writing: All authors; (VII) Final approval of manuscript: All authors. \\ Correspondence to: Dan Sun. Department of Burns, the Affiliated Hospital of Jiangnan University, Wuxi 214000, China. Email: sundan-1981@163.com.
}

Backgroundz Acute myocardial infarction (AMI) is characterized by a critical condition, rapid progression,
and a high fatality rate. Percutaneous coronary intervention (PCI) is the treatment of choice for AMI. The
purpose of this study was to investigate the efficacy of a 7-day gradual early functional exercise program after
PCI in young and middle-aged patients with AMI.
Methods: A total of 106 young and middle-aged patients with AMI at the Affiliated Hospital of Jiangnan
University from May 2018 to May 2019 were selected and divided into 2 groups according to the random
number table method (n=53 per group). Both groups were treated with PCI, and the control group then
received routine functional exercises after surgery, whilst the observation group received a 7-day gradual
early functional exercise program after surgery. Functional exercise compliance, daily life ability before and
after intervention, changes in quality of life, and postoperative complications after 1 year were compared
between the 2 groups.

Results: The functional exercise compliance of the observation group was significantly higher than that of the control group $(\mathrm{P}<0.05)$. The daily living ability of the observation group was also significantly better than that of the control group after the intervention $(\mathrm{P}<0.05)$. The physical activity, emotional response, diet, adverse drug reactions, and anxiety medication scores of the observation group after the intervention were lower than those of the control group $(\mathrm{P}<0.05)$. Furthermore, the postoperative complications and total complication rate after 1 year in the observation group was lower than the control group $(\mathrm{P}<0.05)$.

Conclusions: The application of a 7-day gradual early functional exercise program in young and middleaged patients with AMI after PCI can improve functional exercise compliance, daily life ability, quality of life, and reduce the incidence of complications.

Keywords: 7-day gradual early functional exercise; young and middle-aged; acute myocardial infarction (AMI); percutaneous coronary intervention (PCI); compliance; quality of life; complications

Submitted Oct 12, 2020. Accepted for publication Dec 04, 2020.

doi: 10.21037/apm-20-2243

View this article at: http://dx.doi.org/10.21037/apm-20-2243

\section{Introduction}

Acute myocardial infarction (AMI) is a serious consequence of coronary heart disease, and is characterized by critical illness, rapid progression, and high mortality (1). In recent years, the incidence of AMI among younger people is increasing. Studies have shown that the proportion of young and middle-aged patients with AMI is 3-6\% (2).

Percutaneous coronary intervention (PCI) is a treatment 
method in which transcardiac catheter technology is inserted into narrowed or occluded coronary artery lumen to improve myocardial blood perfusion. PCI is currently the first choice for the clinical treatment of AMI $(3,4)$. The young and middle-aged are the economic pillars of the family unit, and are the backbone of society. Therefore, the treatment of such patients cannot aim at just prolonging life, but to restore heart function as much as possible to reduce loss to the family and society (5). Early progressive exercises, initiated by supervised inpatient training and followed by an unsupervised outpatient program, improved HRQL and functional capacity in patients at low cardiovascular risk who recently experienced an acute myocardial infarction (6). Exercise can improve several cardiovascular and non-cardiovascular parameters, such as glucose metabolism, skeletal muscle function, oxidative stress, vascular function, pulmonary circulation, ischemia-reperfusion lesion, and ventricular remodelling. Rehabilitation exercise is effective in improving functional capacity and quality of life in patients with AMI (7). Young and middle-aged patients after PCI are prone to irritability during rehabilitation exercises due to their eagerness for success, and their compliance is generally poor. Hence, effective intervention measures are needed to improve the effect of their postoperative rehabilitation exercises (8). Therefore, this study analyzed the influence of a 7-day gradual early functional exercise program on daily life ability, quality of life, and postoperative complications at 1 year follow-up in young and middle-aged patients with AMI after PCI, aiming to provide a reference for the clinical development of intervention measures after PCI. We present the following article in accordance with the CONSORT reporting checklist (available at http://dx.doi. org/10.21037/apm-20-2243).

\section{Methods}

\section{Patient selection}

A total of 106 young and middle-aged patients with AMI who underwent surgical treatment at the Affiliated Hospital of Jiangnan University from May 2018 to May 2019 were enrolled in this study. Subjects were divided into an observation group (53 cases) and a control group (53 cases) using the random number table method. Patients who met the following criteria were included in the study: (I) patients met the diagnostic criteria for AMI; (II) patients underwent PCI; (III) aged 18 to 59 years old; (IV) had clear consciousness; (V) had certain language communication ability. Patients were excluded if they met any of the following criteria: (I) cardiac function KilliP class III-IV; (II) comorbidities including serious liver, kidney, lung and other visceral functional diseases, serious cerebrovascular disease, blood system diseases, malignant tumor, or mental disorders; (III) dysfunction in audiovisual integration; (IV) failure to cooperate with postoperative follow-up or midway withdrawal. The study was approved by the Ethics Committee of the Affiliated Hospital of Jiangnan University \{Ethics approval number: [(2018) KY043]\} and conducted in accordance with the Declaration of Helsinki (as revised in 2013). All subjects provided informed consent.

\section{Interventions}

The control group received regular rehabilitation exercises, while the observation group received a 7-day gradual early functional exercise program. The training was performed at the bedside, and the patients were guided by the trained Specialist nurse. The specific plans and processes are shown in Table 1. The program of functional exercise in the observation group was devised by the director of the department, attending physician, provincial and municipal nurses, and nursing supervisors through consultation. The safety of the rehabilitation exercises for middle-aged and young patients with AMI was analyzed by consulting the literature. The time, method, and content of early functional exercises were also clarified, and a specific plan for functional exercises was finally devised.

\section{Evaluation indexes}

\section{Functional exercise compliance}

Complete compliance was defined as exercising on time and according to the doctor's instructions, partial compliance was defined as basically following the doctor's instructions to exercise, but occasionally forgetting to exercise or reduce the intensity of exercise, and non-compliance was defined as not following the doctor's instructions to exercise. In the process of exercise, the instructor scored on the spot. Compliance $=$ complete compliance + partial compliance (9).

\section{Daily life ability}

The functional independence Rating Scale was used to evaluate daily life ability in the 2 groups before and after the intervention. The scale included 6 dimensions of self- 
Table 1 Comparison of the intervention programs in the observation group and the control group

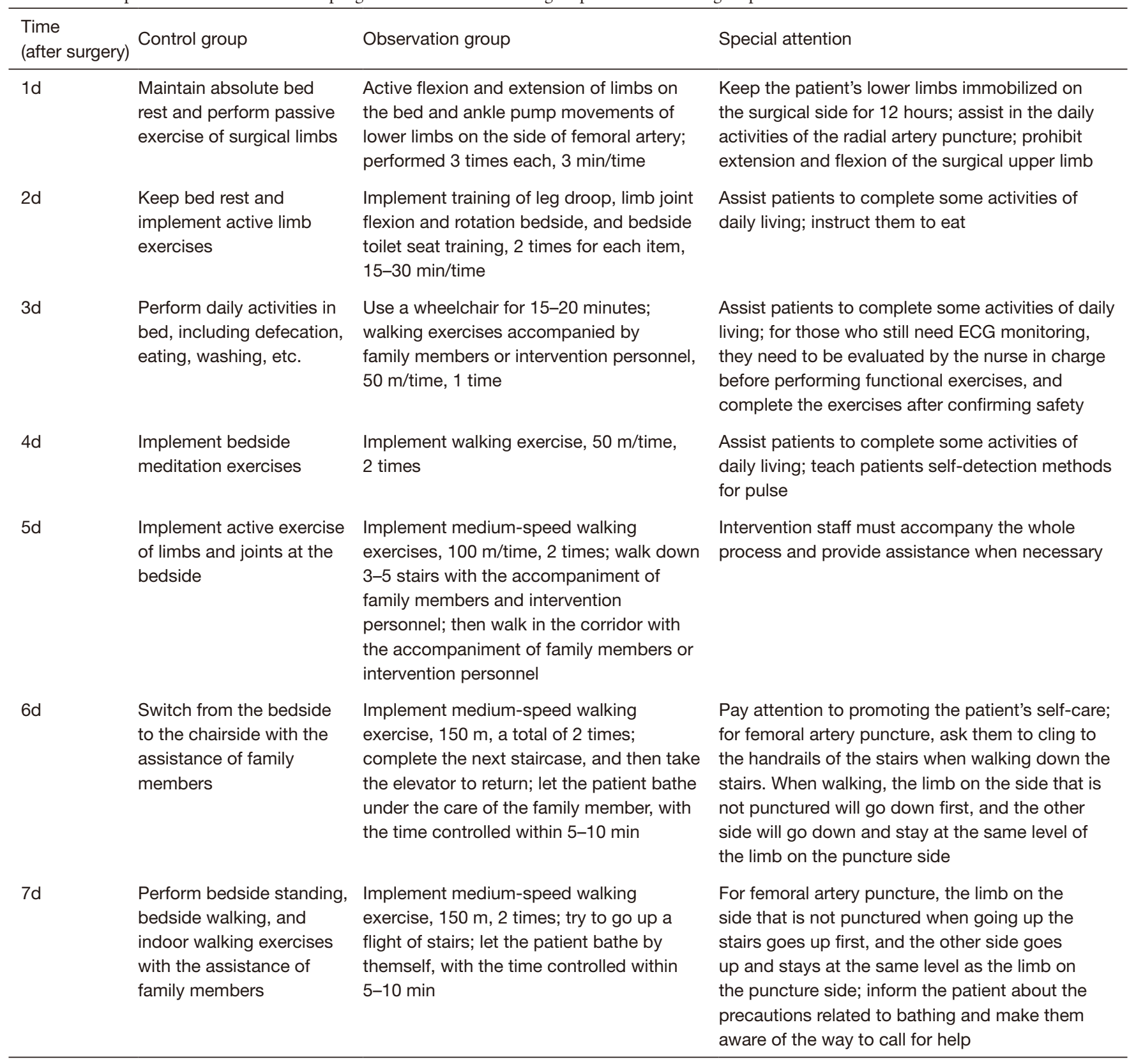

care activity, social cognition ability, sphincter control, communication ability, transfer ability, and walking status. There were 18 items in total, with each item scoring 1-7 points, and the total score was $18-126$ points. Complete independence was defined as 126 points, basic independence was defined as $108-125$ points, conditional independence or extremely light dependence was defined as 90-107 points, mildly dependent was defined as $72-89$ points, moderately dependent was defined as $54-71$ points, severely dependent was defined as 36-53 points, extremely dependent was defined as 19-35 points, and completely dependent was defined as 18 points (10).

\section{Quality of life}

The Myocardial Infarction Dimensional Assessment Scale (MIDAS) was used to evaluate the quality of life of the 2 
groups before and after the intervention. The scale included 7 dimensions of physical activity, emotional response, security, dependence, diet, adverse drug reactions, and anxiety medication. There were 35 items in total, with each item scoring 1-5 points. The lower the score, the better the quality of life (11).

\section{Complications}

The 2 groups were followed up for 1 year after surgery, and the incidence of complications such as angina, arrhythmia, and coronary artery restenosis were statistically analyzed, and the total incidence of complications was finally calculated.

\section{Statistical analyses}

The statistical analysis in this study was performed using SPSS22.0 (SPSS Inc., Chicago, IL, USA). Measurement data were expressed as mean \pm standard deviation and compared using two-tailed $t$-tests. Count data were reported as rates and percentages, and compared using the $\chi^{2}$ test or rank-sum test. $\mathrm{P}<0.05$ was considered statistically significant in this study.

\section{Results}

\section{Baseline characteristics of patients}

The control group included 33 males and 20 females. Their ages ranged from 20 to 58 years old, and the average age was $45.62 \pm 6.39$ years. The observation group included 34 males and 19 females. Their ages ranged from 21 to 59 years old, and the average age was $45.36 \pm 6.51$ years. There were no significant differences in baseline data between the 2 groups $(\mathrm{P}>0.05)$.

\section{Comparison of functional exercise compliance}

The functional exercise compliance of the observation group was significantly higher than that of the control group $\left(\chi^{2}=10.600, \mathrm{P}=0.001\right)$, as shown in Table 2 .

\section{Daily life ability}

Before intervention, there was no statistically significant difference in daily life ability between the observation group and the control group $(Z=0.299, P=0.861)$. After 1 week of intervention, the daily life ability of patients in the observation group was significantly better than that of the control group $(\mathrm{Z}=6.879, \mathrm{P}=0.032)$, as shown in Table 3 .

\section{Quality of life}

Before intervention, there were no statistically significant differences between the observation group and the control group in the scores for physical activity, emotional response, security, dependence, diet, adverse drug reactions, and anxiety medication $(\mathrm{t}=0.363,0.075,0.253,0.200$, $0.830,0.335,0.158$, respectively; $\mathrm{P}=0.717,0.940,0.801$, $0.842,0.408,0.739,0.875$, respectively). After 1 week of intervention, the scores for physical activity, emotional response, diet, adverse drug reactions, and anxiety medication in the observation group were all significantly lower than those of the control group $(t=16.359,11.858$, $10.517,23.162,23.050$, respectively; $\mathrm{P}=0.000,0.000,0.000$, $0.000,0.000$, respectively), as shown in Table 4 .

\section{Complications}

In the control group, 10 cases of angina, 7 cases of arrhythmia, and 7 cases of coronary artery restenosis occurred 1 year after surgery, with a total complication rate of $45.28 \%$. Contrastingly, in the observation group, 4 cases of angina, 1 case of arrhythmia, and no coronary artery restenosis occurred, with a total complication rate of $9.43 \%$. These postoperative complications 1 year after surgery and the total complication rate of the observation group were lower than the control group $\left(\chi^{2}=2.963,3.380\right.$, 5.506, 17.137, respectively; $\mathrm{P}=0.085,0.066,0.019,0.000$, respectively), as shown in Table 5.

\section{Discussion}

In recent years, partly due to the increases in poor lifestyle choices such as lack of exercise, smoking, drinking, poor eating habits, and excessive social interaction, there has been a significant increase in the level of catecholamines in the young and middle-aged population, which in turn stimulates the coronary arteries and induces narrowed coronary arteries spasms. As a result, the incidence of AMI is increasing year by year $(12,13)$. Although PCI can significantly improve the survival rate of young and middleaged patients with AMI, postoperative functional recovery is not optimal. Hence, effective postoperative rehabilitation exercise guidance should be given to such patients (14). A 7 -day gradual early functional exercise program is an early 
Table 2 Comparison of functional exercise compliance between the 2 groups (n, \%)

\begin{tabular}{|c|c|c|c|c|}
\hline Group & Complete compliance & Partial compliance & Non-compliance & Compliance \\
\hline Observation group $(n=53)$ & $38(71.70)$ & $13(24.53)$ & $2(3.77)$ & $51(96.23)$ \\
\hline$\chi^{2}$ value & \multicolumn{4}{|c|}{10.600} \\
\hline$P$ value & \multicolumn{4}{|c|}{0.001} \\
\hline
\end{tabular}

Table 3 Comparison of activities of daily living between the 2 groups before and after intervention (n, \%)

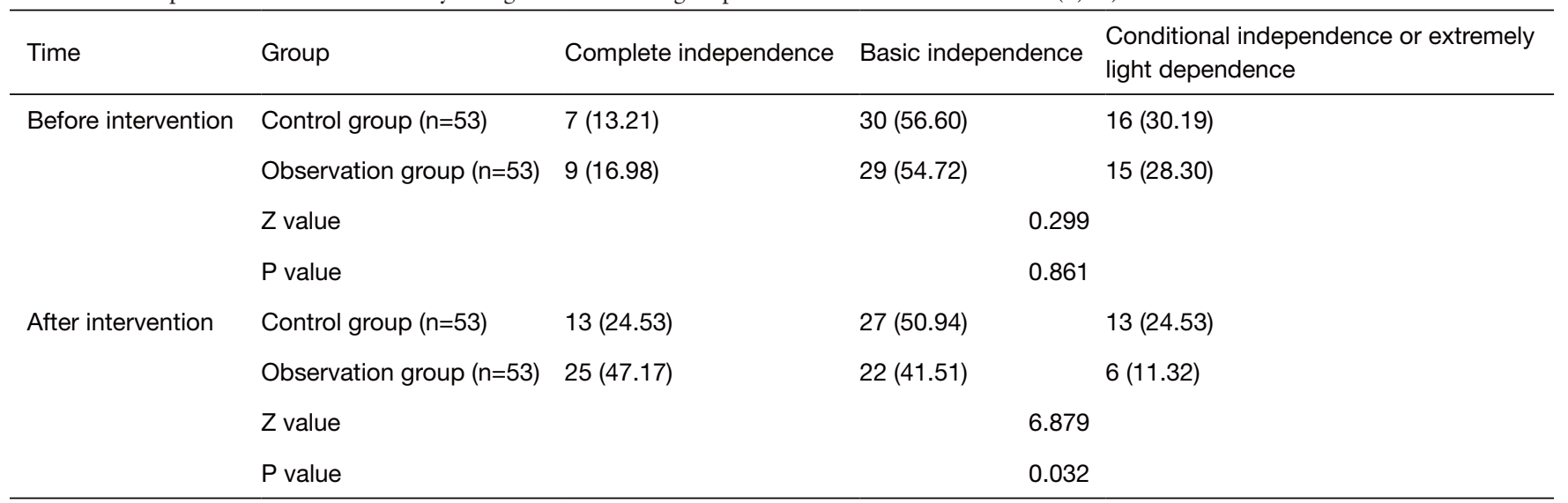

Table 4 Comparison of quality of life between the 2 groups before and after intervention $\left(\bar{x}_{ \pm} \mathrm{SD}\right.$, points)

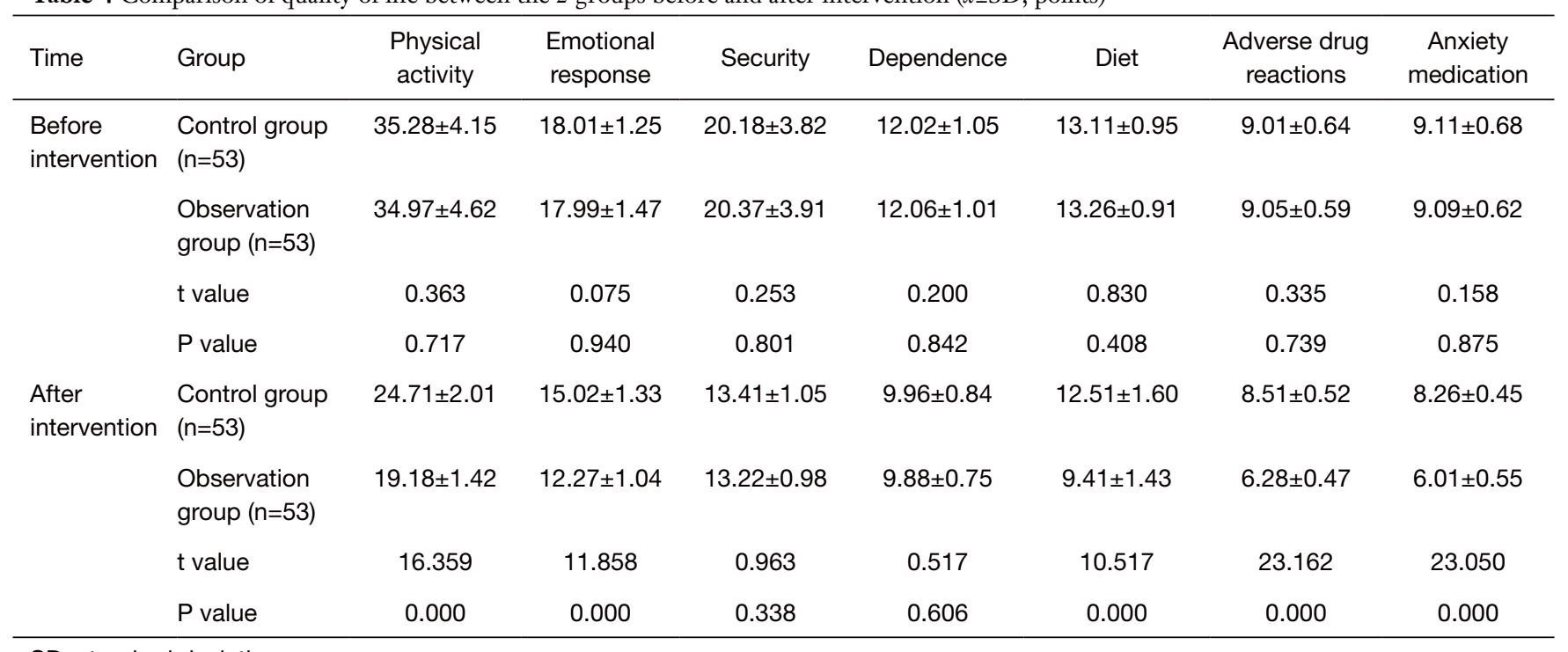

SD, standard deviation. 
Table 5 Comparison of the incidence of complications between the 2 groups (n, \%)

\begin{tabular}{lcccc}
\hline Group & Angina & Arrhythmia & Coronary artery restenosis & Total complications \\
\hline Control group $(\mathrm{n}=53)$ & $10(18.87)$ & $7(13.21)$ & $7(13.21)$ & $24(45.28)$ \\
Observation group $(\mathrm{n}=53)$ & $4(7.55)$ & $1(1.89)$ & $0(0.00)$ & $5(9.43)$ \\
$\chi^{2}$ value & 2.963 & 3.380 & 5.506 & 17.137 \\
$P$ value & 0.085 & 0.066 & 0.019 & 0.000 \\
\hline
\end{tabular}

cardiac exercise measure for young and middle-aged patients with AMI. It follows the principle of gradual and orderly progress to help patients quickly restore cardiac function, so that they can return to society as soon as possible, and take care of their family and societal responsibilities $(15,16)$.

The present findings demonstrated that young and middle-aged patients with AMI in the observation group had significantly higher functional exercise compliance than the control group. This result suggests that the 7-day gradual early functional exercise program can significantly improve patient exercise compliance. There are many factors affecting functional exercise compliance, mainly including complicated rehabilitation exercise content, long rehabilitation exercise cycle, low awareness of rehabilitation exercises, and lack of effective supervision and encouragement (17). The 7-day gradual early functional exercise program is targeted towards the characteristics of the young and middle-aged population. Functional exercise after PCI is divided into days, and the corresponding exercise tasks are completed every day, so that the daily exercise content is clear and easy for patients to master. In addition, the 7-day gradual early functional exercise program clearly corresponds to the postoperative period and the content of the rehabilitation exercise, and provides patients with scientific exercise guidelines, so that patients can complete the rehabilitation exercise with the assistance of the intervention staff, whilst also improving compliance (18).

This study found that the daily living ability of the observation group was better than that of the control group after the intervention. The physical activity, emotional response, diet, adverse drug reactions, and anxiety medication scores of the observation group after the intervention were significantly lower than those of the control group. The results showed that the 7-day gradual early functional exercise program could effectively improve daily life ability and quality of life. The 7-day gradual early functional exercise program is based on the patient's postoperative body recovery, and scientific and gradual early postoperative functional exercise measures are formulated specifically for them. The exercises are devised in line with the recovery progress of the patient's normal cardiac physiological load after surgery, and can promote the patient's coronary blood flow perfusion, establishing collateral circulation as soon as possible $(19,20)$. The 7 -day gradual early functional exercise program can also improve the enthusiasm of the patients' daily life self-care ability, help patients build confidence in overcoming their disease, relieve the psychological burden caused by the disease, and form a good subjective awareness of rehabilitation exercises. In terms of vascular remodeling, the program may also play a positive role in coronary atherosclerotic processes, thereby contributing to improvements in daily life ability and quality of life $(21,22)$.

The results of this study also showed that after 1 year the postoperative complications and the total complication rate of the observation group was significantly lower than the control group. Rehabilitation exercises will not cause complications due to increased physical activity, and instead they prevent the multiple complications that can be caused by prolonged bed rest. The 7-day gradual early functional exercise program is based on the characteristics of sports metabolism in the young and middle-aged population, and includes specially devised scientific rehabilitation exercises to improve the body's anti-thrombotic ability, accelerate vascular remodeling, and reduce the incidence of postoperative angina, arrhythmia, and other complications $(23,24)$.

\section{Conclusions}

In summary, the application of a 7-day gradual early functional exercise program in young and middle-aged patients with AMI after PCI can improve functional exercise compliance, daily life ability, quality of life, and reduce the incidence of complications. While previous studies on this field are limited, and the sample size of this study is not enough. In the next study, we will increase the sample size 
and supplement research indicators to conduct more indepth research on this.

\section{Acknowledgments}

Funding: None.

\section{Footnote}

Reporting Checklist: The authors have completed the CONSORT reporting checklist. Available at http://dx.doi. org/10.21037/apm-20-2243

Data Sharing Statement: Available at http://dx.doi. org/10.21037/apm-20-2243

Conflicts of Interest: All authors have completed the ICMJE uniform disclosure form (available at http://dx.doi. org/10.21037/apm-20-2243). The authors have no conflicts of interest to declare.

Ethical Statement: The authors are accountable for all aspects of the work in ensuring that questions related to the accuracy or integrity of any part of the work are appropriately investigated and resolved. The study was approved by the Ethics Committee of the Affiliated Hospital of Jiangnan University \{Ethics approval number: [(2018) KY043] $\}$ and conducted in accordance with the Declaration of Helsinki (as revised in 2013). All subjects provided informed consent.

Open Access Statement: This is an Open Access article distributed in accordance with the Creative Commons Attribution-NonCommercial-NoDerivs 4.0 International License (CC BY-NC-ND 4.0), which permits the noncommercial replication and distribution of the article with the strict proviso that no changes or edits are made and the original work is properly cited (including links to both the formal publication through the relevant DOI and the license). See: https://creativecommons.org/licenses/by-nc-nd/4.0/.

\section{References}

1. Noureddine S, Dumit NY, Maatouk H. Patients' knowledge and attitudes about myocardial infarction. Nurs Health Sci 2020;22:49-56.

2. Shiyovich A, Axelrod M, Gilutz H, et al. Early Versus Late New-Onset Atrial Fibrillation in Acute Myocardial
Infarction: Differences in Clinical Characteristics and Predictors. Angiology 2019;70:921-8.

3. Worcester MU, Goble AJ, Elliott PC, et al. Mild Depression Predicts Long-Term Mortality After Acute Myocardial Infarction: A 25-Year Follow-Up. Heart Lung Circ 2019;28:1812-8.

4. Yamamoto K, Sakakura K, Akashi N, et al. Clinical outcomes after acute myocardial infarction according to a novel stratification system linked to a rehabilitation program. J Cardiol 2018;72:227-33.

5. Ögmundsdottir Michelsen H, Sjölin I, Schlyter M, et al. Cardiac rehabilitation after acute myocardial infarction in Sweden-evaluation of programme characteristics and adherence to European guidelines: The Perfect Cardiac Rehabilitation (Perfect-CR) study. Eur J Prev Cardiol 2020;27:18-27.

6. Peixoto TC, Begot I, Bolzan DW, et al. Early exercisebased rehabilitation improves health-related quality of life and functional capacity after acute myocardial infarction: a randomized controlled trial. Can J Cardiol 2015 Mar;31(3):308-13.

7. Fontes-Carvalho R, Sampaio F, Teixeira M, et al. The role of a structured exercise training program on cardiac structure and function after acute myocardial infarction: study protocol for a randomized controlled trial. Trials 2015;16:90.

8. Plakht Y, Gilutz H, Arbelle JE, et al. Sex and Ethnic Disparities in Health-Related Outcomes Following Acute Myocardial Infarction in Israel. Isr Med Assoc J 2020;22:303-9.

9. Jiang W, FengM, Gao C, et al. Effect of a nurse-led individualized self-management program for Chinese patients with acute myocardial infarction undergoing percutaneous coronary intervention. Eur J Cardiovasc Nurs 2020;19:320-9.

10. An MY, Li Y, Chen WH, et al. Effects of non-invasive remote ischemic conditioning on rehabilitation after myocardial infarction. Biochem Biophys Res Commun 2017;488:278-84.

11. Jiang Y, Feng YP, Tang LX, et al. The protective role of NR4A3 in acute myocardial infarction by suppressing inflammatory responses via JAK2-STAT3/NF-кB pathway. Biochem Biophys Res Commun 2019;517:697-702.

12. Elbadawi A, Elzeneini $M$, Thakker R, et al. Sex Differences in In-Hospital Outcomes of Transcatheter Mitral Valve Repair (from a National Database). Am J Cardiol 2020;125:1391-7.

13. Irzmański R, Kapusta J, Obrębska-Stefaniak A, et al. 
Cardiac rehabilitation in patients with ST-segment elevation myocardial infarction: can its failure be predicted. Ther Adv Cardiovasc Dis 2017;11:177-84.

14. Protopapas A, Lambrinou E. Cultural factors and the circadian rhythm of ST elevation myocardial infarction in patients in a Mediterranean island. Eur J Cardiovasc Nurs 2019;18:562-8.

15. Tsai YJ, Li MH, Chen CH, et al. Improved oxygen uptake efficiency slope in acute myocardial infarction patients after early phase I cardiac rehabilitation. Int J Rehabil Res 2017;40:215-9.

16. Chien DK, Lee SY, Hung CL, et al. Do patients with non-ST-elevation myocardial infarction without chest pain suffer a poor prognosis. Taiwan J Obstet Gynecol 2019;58:788-92.

17. Melnik MG. Role of age chronotropic criterion in the increase motor activity on an inpatient stage of rehabilitation of geriatric patients with myocardial infarction. Adv Gerontol 2016;29:523-5.

18. Sud K, Haddadin F, Tsutsui RS, et al. Readmissions in STElevation Myocardial Infarction and Cardiogenic Shock (from Nationwide Readmission Database). Am J Cardiol 2019;124:1841-50.

19. Ericsson $M$, Ängerud KH, Brännström $M$, et al.

Cite this article as: Yang Y, Sun L, Feng W, Sun D. Observation of the effect of a 7-day gradual early functional exercise program in middle-aged and young patients with acute myocardial infarction after percutaneous coronary intervention. Ann Palliat Med 2021;10(1):258-265. doi: 10.21037/apm-20-2243
Interaction between tele-nurses and callers with an evolving myocardial infarction: Consequences for level of directed care. Eur J Cardiovasc Nurs 2019;18:545-53.

20. Ding $\mathrm{S}, \mathrm{Xu} \mathrm{S}$, Chen $\mathrm{X}$, et al. Effects of atorvastatin combined with bivalirudin on coagulation function, cardiac function, and inflammatory factors of percutaneous coronary intervention in elderly patients with acute myocardial infarction. Ann Palliat Med 2020;9:1905-11.

21. McCarthy CP, Murphy S, Rehman S, et al. Home-Time After Discharge Among Patients With Type 2 Myocardial Infarction. J Am Heart Assoc 2020;9:e015978.

22. Pandey A, Keshvani N, Vaughan-Sarrazin MS, et al. Evaluation of Risk-Adjusted Home Time After Acute Myocardial Infarction as a Novel HospitalLevel Performance Metric for Medicare Beneficiaries. Circulation 2020;142:29-39.

23. Bårdsgjerde EK, Landstad BJ, Hole T, et al. Nurses' perceptions of patient participation in the myocardial infarction pathway. Nurs Open 2020;7:1606-15.

24. Boinska J, Koziński M, Kasprzak M, et al. Diurnal variations in tissue factor and tissue factor pathway inhibitor concentrations in relation to on-treatment platelet reactivity: an analysis of patients with acute myocardial infarction. Platelets 2020;31:877-83. 